\title{
The impact of selective serotonin receptor inhibitors on post-endoscopic sphincterotomy bleeding, alone or with concurrent aspirin or nonsteroidal anti-inflammatory drugs
}

\author{
Panagiotis Katsinelos ${ }^{\mathrm{a}, \mathrm{b}}$, Georgia Lazaraki ${ }^{\mathrm{b}}$, Sotiris Anastasiadis ${ }^{\mathrm{b}}$, Grigoris Chatzimavroudisa, \\ Taxiarchis Katsinelos c, Sotiris Terzoudis ${ }^{b}$, Anthi Gatopoulou ${ }^{b}$, Michael Doulberis ${ }^{b}$, \\ Apostolis Papaefthimiou $^{b}$, Jannis Kountouras ${ }^{b}$ \\ G. Gennimatas General Hospital; Ippokration Hospital, Aristotle University Thessaloniki, Thessaloniki Greece
}

\section{Abstract}

\begin{abstract}
Background Observationalstudieshave shown an increased risk of uppergastrointestinalbleeding in users of selective serotonin receptor inhibitors (SSRIs). We retrospectively investigated the impact of SSRIs, alone or combined with aspirin (ASA) or nonsteroidal anti-inflammatory drugs (NSAIDs), on the incidence of post-endoscopic sphincterotomy (post-ES) bleeding.
\end{abstract}

Methods A total of 3058 patients were included. Of these, 457 patients received SSRIs, alone or plus ASA or NSAIDs, until the day of ES (SSRIs group), while 2659 patients (non SSRIs group) had never been on SSRIs $(n=1925)$, though some had been on ASA $(n=613)$ or NSAIDS $(n=121)$. Patient assessment included indication for endoscopic retrograde cholangiopancreatography (ERCP), comorbid diseases, detailed drug history before and after ES, procedural details, and risk factors for post-ES bleeding. Primary outcome was defined as the incidence, type and severity of post-ES bleeding.

Results There was no statistical difference in age, sex, indication for ERCP, comorbid diseases, technical characteristics or results of therapeutic ERCP between the 2 groups. The incidence of post-ES bleeding was $3.9 \%$ in the SSRIs group and 3\% in the non SSRIs group, a difference not statistically significant $(\mathrm{P}=0.754)$. Likewise, there was no difference in type $(\mathrm{P}=0.145)$ or severity of bleeding $(\mathrm{P}=0.754)$ between the 2 groups. Multivariate analysis showed the precut technique as the only independent risk factor for post ES hemorrhage (odds ratio 2.56, 95\% confidence interval 1.23-3.63; $\mathrm{P}=0.001$ ).

Conclusion This study found that SSRIs, alone or combined with ASA or NSAIDs, had no influence on the incidence or the severity of post-ES bleeding.

Keywords Endoscopic sphincterotomy, bleeding, selective serotonin receptor inhibitors, aspirin, nonsteroidal anti-inflammatory drugs

Ann Gastroenterol 2019; 32 (6): 1-6
Department of a Endoscopy, G. Gennimatas General Hospital (Panagiotis Katsinelos, Grigoris Chatzimavroudis); ${ }^{\text {EEndoscopy, }}$ Ippokration Hospital, Aristotle University Thessaloniki (Panagiotis Katsinelos, Georgia Lazaraki, Sotiris Anastasiadis, Sotiris Terzoudis, Anthi Gatopoulou, Michael Doulberis, Apostolis Papaefthimiou,

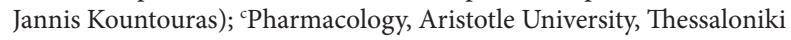
(Taxiarchis Katsinelos), Thessaloniki, Greece

\section{Conflict of Interest: None}

Correspondence to: Panagiotis Katsinelos, MD, PhD, Associate Professor of Gastroenterology, Department of Endoscopy, Ippokration Hospital, School of Medicine, Faculty of Health Sciences, Aristotle University of Thessaloniki, Greece, e-mail: gchatzimav@yahoo.gr

Received 18 April 2019; accepted 18 July 2018; published online 8 October 2019

DOI: https://doi.org/10.20524/aog.2019.0425

\section{Introduction}

Endoscopic retrograde cholangiopancreatography (ERCP) in the modern era is mainly therapeutic, while endoscopic sphincterotomy (ES) remains the cornerstone for a successful procedure [1]. The main complications of therapeutic ERCP include post-ERCP pancreatitis, post-ES bleeding, perforation, cholangitis, and adverse cardiopulmonary events [1-4]. The incidence of post-ES bleeding depends on the definition applied, and ranges from $2-7 \%$ [1-3]. All recently published guidelines [5-7] on antiplatelet use during endoscopic procedures recommend withholding thienopyridines while continuing aspirin (ASA) and nonsteroidal anti-inflammatory drugs (NSAIDs) in patients undergoing ES. 
Selective serotonin reuptake inhibitors (SSRIs) have a well-known antiplatelet activity [8]. Platelets release serotonin in response to vascular injury; this triggers vasoconstriction and platelet aggregation, resulting in hemostasis [8]. Long-term treatment with SSRIs upregulates the expression of glucosesynthase kinase 3- $\beta$ (GSK3B) on platelets [9]. GSK3B acts as a negative regulator of platelets and thrombosis and might contribute to bleeding risk with SSRI use [9]. Thus, SSRIs, either alone or in combination with ASA or NSAIDs, have been associated with an increased risk of upper gastrointestinal bleeding, post-surgery or after percutaneous endoscopic gastrostomy (PEG) [10-14].

In the literature, there is only one recent small retrospective cohort study that investigated the influence of SSRIs and serotonin-norepinephrine reuptake inhibitors on immediate and delayed bleeding post-ES and found no association [15]. The aim of our study was to investigate the possible impact of SSRIs, alone or combined with ASA or NSAIDs, on the incidence of post-ES bleeding.

\section{Patients and methods}

This study was conducted after obtaining necessary approval from the Institutional Review Board of our hospital. We retrospectively analyzed the medical records of all patients who underwent therapeutic ERCP between January 2000 and December 2017. The patients were categorized according to whether they continued to take SSRIs, alone or with ASA or NSAIDS, until the day of the procedure, or had never been on SSRIs. Patients' SSRIs and ASA/NSAIDs dosages were not included, since the aim of the study was to analyze the bleeding risk with the aforementioned therapies. Data comprised demographics, clinical history of medical comorbidities, including renal, cardiovascular and hepatic issues, coagulation disorders, bleeding disorders, coagulation profile, blood results, endoscopic findings (periampullary diverticulum, naïve papilla), procedure details (conventional ES or precut technique, mechanical lithotripsy, type of stent placed) and complete drug history, focusing on the use of SSRIs alone, or combined with ASA or NSAIDs, at the time of the procedure. All ESs were performed by an experienced pancreatobiliary endoscopist (PK). A microprocessorcontrolled ERBE electrosurgical generator was used and all ES procedures were performed with endocut current (120 W cut; $60 \mathrm{~W}$ coagulation, ERBE USA, Atlanta, Georgia, USA). Minimal, self-limited bleeding during the procedure was not recorded as bleeding.

The type of post-ES bleeding was classified as intraprocedural, early (bleeding episode within $24 \mathrm{~h}$ after ES but not evident during procedure), or delayed (bleeding episode occurred up to 20 days after ES and manifested as melena, hematemesis or hematochezia associated with a decrease in hemoglobin level). Bleeding severity was defined by consensus criteria [16] graded as follows: 1) mild, clinical evidence with a decrease in hemoglobin level ( $>3 \mathrm{~g} / \mathrm{dL}$ ), with blood transfusion not required; 2 ) moderate, transfusion required ( $\leq 4$ units) but not angiographic intervention or surgery; or 3 ) severe, transfusion of 5 or more units of blood or the need for surgical or angiographic intervention.

Endoscopic hemostasis was carried out with injection of 1:10000 epinephrine solution, or with coagulation current applied by the apex of the sphincterotome or the tip of a polypectome snare, and rarely by a heater probe. The primary endpoint of the study was to investigate the impact of SSRIs, alone or with concomitant ASA or NSAID use, on the incidence, type and severity of post-ES bleeding.

\section{Statistical analysis}

Categorical variables were analyzed using the chi-square and Fisher's exact tests, as appropriate, while continuous variables were expressed as mean \pm standard deviation and analyzed using Student's $t$-test. Possible risk factors for post-ES bleeding were examined using univariate and multivariate analyses and the odds ratio (OR) and 95\% confidence interval (CI) were calculated using a logistic regression method. Statistical significance was set at $\mathrm{P}<0.05$. Statistical analysis was performed using the Statistical Package for Social Sciences (SPSS, version 19.0, Chicago, IL, USA).

\section{Results}

Using the database of our departments we identified 12,204 patients who underwent therapeutic ERCP from 2000-2017; 3058 of these were included in the final analysis. At the time of the procedure, 322 patients were taking SSRIs, 37 patients SSRIs plus NSAIDs and 98 patients SSRIs plus ASA therapy (SSRIs group) (Table 1). The control group (non-SSRIs) comprised 1925 patients who had not received SSRIs, of whom 613 and 121 patients had taken ASA or NSAIDs, respectively (Table 1 ). The patient characteristics of the total cohort are presented in Table 1. No statistically significant difference was found between the 2 groups regarding sex distribution, indication for ERCP, or comorbid diseases; however, patients were younger in the non-SSRIs group $(\mathrm{P}<0.05) \quad($ Table 1$)$. The technical characteristics and the results of therapeutic ERCP are shown in Table 2, indicating no statistically significant difference between the 2 groups.

The incidence of post-ES bleeding was 3.9\% in the SSRIs group and $3 \%$ in the non-SSRIs group, a non-significant difference $(\mathrm{P}=0.754)$. Likewise, there were no differences regarding the type $(\mathrm{P}=0.145)$ or severity $(\mathrm{P}=0.623)$ of post-ES bleeding between the 2 groups (Table 3 ).

Table 4 shows the association between the type of antiplatelet drug and the severity of post-ES bleeding.

Univariate analysis yielded the following significant risk factors for post-ES bleeding: precut sphincterotomy (OR 2.56, 95\%CI 1.23-363; $\mathrm{P}=0.001$ ), anticoagulation use (OR 
Table 1 Clinical characteristics of patients

\begin{tabular}{lccc}
\hline Characteristics & $\begin{array}{c}\text { Patients on SSRIs, SSRIs plus Aspirin, } \\
\text { or SSRIs plus NSAIDs (SSRI group) }\end{array}$ & Patients not taking SSRIs (non-SSRI group) & P-value \\
\hline No. patients & 457 & 2601 & $<0.05$ \\
Age (years) (mean \pm SD) & $73.25+26.86$ & $62.35+33.53$ & 0.621 \\
Male/Female & $216 / 241$ & $1138 / 1463$ &
\end{tabular}

\begin{tabular}{lccc}
\hline Indication for ERCP & & & \\
Choledocholithiasis & $321(70.24)$ & $2031(78.09)$ & 0.249 \\
\hline Cholangitis & $8(1.75)$ & $47(1.81)$ & 0.943 \\
\hline Biliary or pancreatic cancer & $47(10.28)$ & $211(8.11)$ & 0.467 \\
\hline Acute pancreatitis & $21(4.6)$ & $98(3.77)$ & 0.333 \\
Biliary leak & $18(3.94)$ & $77(2.96)$ & 0.216 \\
Others & $42(9.19)$ & $137(5.27)$ & 0.371 \\
\hline
\end{tabular}

\section{Comorbid diseases}

\begin{tabular}{lccc} 
Heart disease & $211(46.17)$ & $1370(52.67)$ & 0.788 \\
Pulmonary disease & $83(18.16)$ & $334(12.84)$ & 0.231 \\
\hline Previous stroke & $8(1.75)$ & $34(1.31)$ & 0.218 \\
Hypertension & $254(55.57)$ & $1240(47.67)$ & 0.312 \\
Diabetes & $115(25.16)$ & $513(19.72)$ & 0.193 \\
Cirrhosis & $7(1.53)$ & $23(0.88)$ & 0.633 \\
\hline
\end{tabular}

\begin{tabular}{lccc}
\hline SSRIs alone & $322(70.46)$ & 0 & $121(4.65)$ \\
\hline NSAIDs & $37(8.1)$ & $613(23.56)$ & 0.143 \\
\hline Aspirin & $98(21.44)$ & $63(2.42)$ & 0.854 \\
\hline Anticoagulation treatment & $19(4.16)$ & & 0.298 \\
\hline Values are given as n (\%) unless otherwise indicated & &
\end{tabular}

1.89, 95\%CI 1.23-3.63; $\mathrm{P}=0.022$ ), ASA use (OR 2.05, 95\%CI 1.14-3.94; $\mathrm{P}=0.018$ ), and NSAID use (OR 1.72, 95\%CI 1.023.03; $\mathrm{P}=0.037$ ) (Table 5). However, multivariate analysis demonstrated only precut sphincterotomy as a significant risk factor (OR 1.98, 95\%CI 1.16-4.21; $\mathrm{P}=0.001$ ) for post-ES hemorrhage.

\section{Discussion}

Since its introduction, ERCP continues to be the main procedure for the endoscopic treatment of pancreatobiliary diseases $[17,18]$. Post-ES bleeding is a well-known complication of ES, and therefore ES is stratified as a high-risk procedure. The current guidelines of all gastrointestinal societies [5-7] include recommendations for the use of antiplatelet drugs (ASA, NSAIDs, aminopyridines) during ES, but surprisingly they do not mention the antiplatelet effect of SSRIs on post-ES bleeding.
In this retrospective cohort study, we found no statistically significant difference in post-ES bleeding associated with SSRI use alone, or with concurrent ASA or NSAIDs. Indeed, there were no differences in the incidence, type or severity of post-ES bleeding between the 2 groups studied. Specifically, the incidence of post-ES bleeding was 3.9\% in the SSRIs group and 3\% in the non SSRIs group, consistent with other published studies [1-3]. We also found that SSRIs, alone or with concurrent ASA or NSAIDs, were not associated with an increased risk of post-ES bleeding (Table 3). Moreover, there was no difference in the type or severity of post-ES hemorrhage, and no statistical difference in the risk factors for post-ES bleeding between the 2 groups (Table 4). However, multivariate analysis of risk factors, including SSRIs and SSRIs plus ASA or NSAIDs, showed that precut sphincterotomy was the only studied risk factor for post-ES bleeding. Precut sphincterotomy per se has been reported to have high bleeding rates, reaching up to $27 \%$ in some series $[19,20]$. Comparable results regarding the effect 
Table 2 Technical characteristics and result of therapeutic ERCP

\begin{tabular}{|c|c|c|c|}
\hline \multirow[t]{2}{*}{ Characteristics } & $\begin{array}{l}\text { SSRI } \\
\text { group }\end{array}$ & $\begin{array}{l}\text { Non-SSRI } \\
\text { group }\end{array}$ & P-value \\
\hline & $(\mathrm{N}=457)$ & $(\mathrm{N}=2601)$ & \\
\hline $\begin{array}{l}\text { Periampullary } \\
\text { diverticulum }\end{array}$ & $89(19.47)$ & $548(21.07)$ & 0.843 \\
\hline Conventional ES & $401(87.74)$ & $2300(88.43)$ & 0.352 \\
\hline Precut technique & $48(10.5)$ & $284(10.91)$ & 0.510 \\
\hline $\begin{array}{l}\text { Visible bleeding } \\
\text { during ES }\end{array}$ & $30(6.56)$ & $152(5.84)$ & 0.482 \\
\hline $\begin{array}{l}\text { Cannulation } \\
\text { failure }\end{array}$ & $8(1.75)$ & $17(0.65)$ & 0.132 \\
\hline Choledocholithiasis & $321(70.24)$ & $2031(78.09)$ & 0.249 \\
\hline $\begin{array}{l}\mathrm{CBD} \\
\text { diameter }(\mathrm{mm})\end{array}$ & $11.54+2.54$ & $10.36+2.61$ & 0.143 \\
\hline Number of stones & $2.11+0.64$ & $1.97+0.73$ & 0.426 \\
\hline $\begin{array}{l}\text { Size of } \\
\text { stones }(\mathrm{mm})\end{array}$ & $10.83+2.54$ & $10.32+3.17$ & 0.735 \\
\hline $\begin{array}{l}\text { CBD clearance of } \\
\text { stones }\end{array}$ & $301(93.77)$ & $1932(95.13)$ & 0.793 \\
\hline Basket or balloon & $170(52.96)$ & $1105(54.14)$ & 0.513 \\
\hline $\begin{array}{l}\text { Mechanical } \\
\text { lithotripsy }\end{array}$ & $65(20.25)$ & $394(19.4)$ & 0.448 \\
\hline Stent placement & $17(5.26)$ & $127(6.25)$ & 0.381 \\
\hline
\end{tabular}

Values are given as $\mathrm{n}(\%)$ or mean $\pm \mathrm{SD}$.

$E S$, endoscopic sphincterotomy; CBD, common bile duct

Table 3 Incidence and severity of post-ES bleeding

\begin{tabular}{|c|c|c|c|}
\hline \multirow[t]{2}{*}{ Post-ES bleeding } & Group A & Group B & P-value \\
\hline & N (\%) & N (\%) & \\
\hline $\begin{array}{l}\text { Total post-ES } \\
\text { bleeding }\end{array}$ & 18 & 79 & 0.754 \\
\hline $\begin{array}{l}\text { Type of bleeding } \\
\text { Intraprocedural } \\
\text { Early } \\
\text { Delayed }\end{array}$ & $\begin{array}{c}13(72.22) \\
4(22.22) \\
1(5.55)\end{array}$ & $\begin{array}{c}64(81.01) \\
11(13.92) \\
4(5.06)\end{array}$ & 0.145 \\
\hline $\begin{array}{l}\text { Severity of bleeding } \\
\text { Mild } \\
\text { Moderate } \\
\text { Severe } \\
\text { Death }\end{array}$ & $\begin{array}{c}11(61.11) \\
5(27.78) \\
2(11.11) \\
0\end{array}$ & $\begin{array}{c}55(69.62) \\
19(24.05) \\
5(6.33) \\
0\end{array}$ & 0.623 \\
\hline
\end{tabular}

ES, endoscopic sphincterotomy

of SSRIs on post-ES bleeding were also reported in a recent small retrospective cohort study [15].

Our findings contradict those of other studies that found SRRIs were associated with a greater incidence of bleeding manifestations, including upper and lower gastrointestinal tract hemorrhage [13,14,21-23]. Indeed, in a meta-analysis of 15 case-control studies and four cohort studies, SSRIs alone were found to be associated with a modest increase
Table 4 Type of antiplatelet drug and severity of post-endoscopic bleeding

\begin{tabular}{lc}
\hline Type of antiplatelet drug/drugs & $(\mathrm{N}=53)$ \\
\hline Aspirin alone & 27 \\
Mild & 19 \\
Moderate & 6 \\
Severe & 1 \\
Death & 0 \\
NSAIDs alone & 8 \\
Mild & 4 \\
Moderate & 3 \\
Severe & 1 \\
Death & 0 \\
SSRIs+aspirin & 9 \\
Mild & 6 \\
Moderate & 2 \\
Severe & 1 \\
Death & 0 \\
SSRIs+NSAIDs & 6 \\
Mild & 3 \\
Moderate & 2 \\
Severe & 1 \\
Death & 0 \\
SSRIs alone & 3 \\
Mild & 2 \\
Moderate & 1 \\
Severe & 0 \\
Death & 0 \\
\hline SSRIs serotoin & 3 \\
\hline
\end{tabular}

SSRIs, serotonin selective reuptake inhibitors; NSAIDs, nonsteroidal anti-inflammatory drugs

in the risk of upper gastrointestinal bleeding (OR 1.68, 95\%CI 1.13-2.50); this risk, however, was significantly elevated when SSRI medications were used in combination with NSAIDs (OR 4.25, 95\%CI 2.82-6.42) [13]. In another retrospective study, the use of SSRIs $24 \mathrm{~h}$ prior to PEG was associated with an increased risk of bleeding (OR 4.1, 95\%CI 1.1-13.4; $\mathrm{P}=0.04)$ [14].

SSRIs are used as first-line pharmacotherapy for depression and are approved for the treatment of various psychogenic disorders because they have a favorable safety profile compared with older-generation antidepressants [13]; however, they are known to demonstrate antiplatelet activity [8] and to act on reduction in platelet/endothelial activation synergistically with concurrent antiplatelet drugs, such as ASA, NSAIDs and aminopyridines [13]. SSRI use has been reported to be associated with an increased risk of upper gastrointestinal bleeding [12] and post-procedure bleeding after PEG [14]. Moreover, a retrospective study of more than 530,000 adults who underwent major surgery showed that SSRI use increased the rate of late post-surgical bleeding slightly but significantly. Additionally, a recent meta-analysis showed that the concomitant use of SSRIs and NSAIDs or ASA is associated with a greater increase in the risk of upper gastrointestinal bleeding than the use of either type of drug alone [9]. One possible explanation for these contradicting results is that during ES a cutting current that also coagulates 
Table 5 Risk factors for post-endoscopic sphincterotomy bleeding

\begin{tabular}{|c|c|c|c|c|c|c|}
\hline \multirow[t]{2}{*}{ Risk factors } & \multicolumn{3}{|c|}{ Univariate analysis } & \multicolumn{3}{|c|}{ Multivariate analysis } \\
\hline & P-value & OR & $95 \% \mathrm{CI}$ & P-value & OR & $95 \% \mathrm{CI}$ \\
\hline Anticoagulation within 3 days of ES & 0.022 & 1.89 & $1.23-3.63$ & 0.08 & 1.23 & $0.65-2.74$ \\
\hline Cholangitis & 0.654 & 1.12 & $0.45-1-93$ & & & \\
\hline Cirrhosis & 0.074 & 1.42 & $0.76-2.77$ & & & \\
\hline Dilated CBD & 0.318 & 0.75 & $0.32-1.84$ & & & \\
\hline Periampullary diverticulum & 0.523 & 1.08 & $0.56-2.04$ & & & \\
\hline Precut sphincterotomy & $<0.001$ & 2.56 & $1.23-4.74$ & $<0.001$ & 1.98 & $1.16-4.21$ \\
\hline Visible bleeding during ES & 0.181 & & & & & \\
\hline SSRIs & 0.137 & 0.86 & $0.25-2.67$ & & & \\
\hline Aspirin & 0.018 & 2.05 & $1.14-3.94$ & 0.074 & 1.67 & $0.67-2.46$ \\
\hline NSAIDs & 0.037 & 1.72 & $1.02-3.03$ & 0.167 & 1.19 & $0.58-2.19$ \\
\hline SSRIs + aspirin & 0.041 & 1.68 & $1.15-2.77$ & 0.94 & 1.47 & $0.87-2.31$ \\
\hline SSRIs + NSAIDs & 0.064 & 1.45 & $0.48-2.28$ & & & \\
\hline
\end{tabular}

ES, endoscopic sphincterotomy; CBD, common bile duct; SSRIs, serotonin selective reuptake inhibitors; NSAIDs, nonsteroidal anti-inflammatory drugs; OR, odds ratio; CI, confidence interval

is used, thus ablating the exposed vessels; it is well known that endocut mode supplies electrosurgical current in intermittent pulses, automatically reducing its voltage after the starting phase and releasing a "soft" coagulation current, insufficient to cut tissue, between pulses of cut current [24]. This is different to the procedure for PEG and upper and lower gastrointestinal bleeding, where no thermic method is applied to the exposed vessels. Indeed our results are in accordance with a retrospective cohort study regarding the impact of SSRIs on post-ES bleeding [15].

It is important to note that clinicians must consider the risk-benefit ratio of discontinuing an SSRI before an elective operative procedure. Discontinuing SSRI medications may result in discontinuation syndrome, symptom recrudescence, or relapse of depression [23,25]. Although the morbidity might be greater in patients under SSRI treatment, the mortality is still low [11].

Our study presents important limitations. No definite guidelines can be derived from this retrospective study, while all procedures were performed by an experienced pancreatobiliary endoscopist and this may have affected the generalizability of our results. It is well-known that a low case volume [26-28] on the part of the endoscopist may reflect less precise control of the incision, increasing the "zipper" cut phenomenon and therefore the post-ES bleeding. In addition, the sample size of SSRIs users was small, exposing our analysis to the possibility of type II error. Although we had 457 SSRIs users in our study and found an incidence of post-ES bleeding of $3.9 \%$ in the SSRIs group and 3\% in the non SSRIs group, the necessary number of patients enrolled in each arm to detect a statistically significant difference would be approximately 5300 . We consider that a prospective, appropriately powered trial is indicated to further investigate the impact of SSRIs, alone or in combination with ASA and NSAIDs, on post-ES bleeding.

\section{Summary Box}

\section{What is already known:}

- Selective serotonin reuptake inhibitors (SSRIs) have a well-known antiplatelet activity

- SSRIs, either alone or in concomitant with aspirin (ASA) or nonsteroidal anti-inflammatory drugs (NSAID), have been associated with an increased risk of upper gastrointestinal bleeding, post-surgery or after percutaneous endoscopic gastrostomy (PEG)

\section{What the new findings are:}

- No statistically significant difference was observed in post-ES bleeding with SSRIs, alone or with concurrent ASA or NSAIDs, in comparison with patients not taking SSRIs

- The type and severity of post-ES hemorrhage did not seem to be influenced by SSRI use

- Our results contradict data from upper gastrointestinal bleeding after PEG; we speculate that this can be explained by the fact that during ES a cutting current that also coagulates is used, thus ablating the exposed vessels

In conclusion, our study found no evidence for an effect of SSRIs, alone or in combination with ASA or NSAIDs, on postES bleeding, though powerful prospective multicenter studies are needed to confirm our results. 


\section{References}

1. Freeman ML. Complications of endoscopic retrograde cholangiopancreatography: avoidance and management. Gastrointest Endosc Clin N Am 2012;22:567-586.

2. Wang P, Li ZS, Liu F, et al. Risk factors for ERCP-related complication: a prospective multicenter study. Am J Gastroenterol 2009;104:31-40.

3. Ferreira LE, Baron TH. Post-sphincterotomy bleeding: who, what, when, and how. Am J Gastroenterol 2007;102:2850-2858.

4. Talukdar R. Complications of ERCP. Best Pract Res Clin Gastroenterol 2016;30:793-805.

5. Acosta RD, Abraham NS, Chandrasekhara V, et al; ASGE Standards of Practice Committee. The management of antithrombotic agents for patients undergoing GI endoscopy. Gastrointest Endosc 2016;83:3-16.

6. Veitch AM, Vanbiervliet G, Gershlick AH, et al. Endoscopy in patients on antiplatelet or anticoagulant therapy, including direct oral anticoagulants: British Society of Gastroenterology and European Society of Gastrointestinal Endoscopy guidelines. Endoscopy 2016;48:385-402.

7. Chan FKL, Goh KL, Reddy N, et al. Management of patients on antithrombotic agents undergoing emergency and elective endoscopy: joint Asian Pacific Association of Gastroenterology (APAGE) and Asian Pacific Society for Digestive Endoscopy (APSDE) practice guidelines. Gut 2018;67:405-417.

8. de Abajo FJ. Effects of selective serotonin reuptake inhibitors on platelet function: mechanisms, clinical outcomes and implications for use in elderly patients. Drugs Aging 2011;28:345-367.

9. Joaquim HP, Talib LL, Forlenza OV, Diniz BS, Gattaz WF. Longterm sertraline treatment increases expression and decreases phosphorylation of glycogen synthase kinase-3B in platelets of patients with late-life major depression. J Psychiatr Res 2012;46:1053-1058.

10. Jiang HY, Chen HZ, Hu XJ, et al. Use of selective serotonin reuptake inhibitors and risk of upper gastrointestinal bleeding: a systematic review and meta-analysis. Clin Gastroenterol Hepatol 2015; 13:42-50

11. Auerbach AD, Vittinghoff E, Maselli J, Pekow PS, Young JQ, Lindenauer PK. Perioperative use of selective serotonin reuptake inhibitors and risks for adverse outcomes of surgery. JAMA Intern Med 2013;173:1075-1081.

12. de Jong JC, van den Berg PB, Tobi $\mathrm{H}$, de Jong-van den Berg LT. Combined use of SSRIs and NSAIDs increases the risk of gastrointestinal adverse effects. $\mathrm{Br} \mathrm{J}$ Clin Pharmacol 2003;55:591-595.

13. Anglin R, Yuan Y, Moayyedi P, Tse F, Armstrong D, Leontiadis GI. Risk of upper gastrointestinal bleeding with selective serotonin reuptake inhibitors with or without concurrent nonsteroidal antiinflammatory use: a systematic review and meta-analysis. Am J Gastroenterol 2014;109:811-819.

14. Richter JA, Patrie JT, Richter RP, et al. Bleeding after percutaneous endoscopic gastrostomy is linked to serotonin reuptake inhibitors, not aspirin or clopidogrel. Gastrointest Endosc 2011;74:22-34.

15. Yadav D, Vargo J, Lopez R, Chahal P. Does serotonin reuptake inhibitor therapy increase the risk of post-sphincterotomy bleeding in patients undergoing endoscopic retrograde cholangiopancreatography? World J Gastrointest Endosc 2017;9:171-176.

16. Cotton PB, Lehman G, Vennes J, et al. Endoscopic sphincterotomy complications and their management: an attempt at consensus. Gastrointest Endosc 1991;37:383-393.

17. Kawai K, Akasaka Y, Murakami K, Tada M, Koli Y. Endoscopic sphincterotomy of the ampulla of Vater. Gastrointest Endosc 1974;20:148-151.

18. Akaraviputh T, Lohsiriwat V, Swangsri J, Methasate A, Leelakusolvong S, Lertakayamanee N. The learning curve for safety and success of precut sphincterotomy for therapeutic ERCP: a single endoscopist's experience. Endoscopy 2008;40:513-516.

19. Freeman ML. Understanding risk factors and avoiding complications with endoscopic retrograde cholangiopancreatography. Curr Gastroenterol Rep 2003;5:145-153.

20. Classen M, Demling L. Endoscopic sphincterotomy of the papilla of vater and extraction of stones from the choledochal duct (author's transl). Dtsch Med Wochenschr 1974;99:496-497.

21. Cheng YL, Hu HY, Lin XH, et al. Use of SSRI, but not SNRI, increased upper and lower gastrointestinal bleeding: a nationwide population-based cohort study in Taiwan. Medicine (Baltimore) 2015;94:e2022.

22. Patel H, Gaduputi V, Sakam S, Kumar K, Chime C, Balar B. Serotonin reuptake inhibitors and post-gastrostomy bleeding: reevaluating the link. Ther Clin Risk Manag 2015;11:1283-1289.

23. Roose SP, Rutherford BR. Selective serotonin reuptake inhibitors and operative bleeding risk: a review of the literature. J Clin Psychopharmacol 2016;36:704-709.

24. Oka Y, Okamoto K, Kawashita N, Shirakuni Y, Takagi T. Metaanalysis of the risk of upper gastrointestinal hemorrhage with combination therapy of selective serotonin reuptake inhibitors and non-steroidal anti-inflammatory drugs. Biol Pharm Bull 2014;37:947-953.

25. Saad J, Ziahosseini K, Wearne G, Ali S, Malhotra R. Selective serotonin reuptake inhibitors and perioperative bleeding in endoscopic dacryocystorhinostomy. Eye (Lond) 2014;28:1268-1269.

26. Keswani RN, Qumseya BJ, O'Dwyer LC, Wani S. Association between endoscopist and center endoscopic retrograde cholangiopancreatography volume with procedure success and adverse outcomes: a systematic review and meta-analysis. Clin Gastroenterol Hepatol 2017;15:1866-1875.e3.

27. Kapral C, Duller C, Wewalka F, Kerstan E, Vogel W, Schreiber F. Case volume and outcome of endoscopic retrograde cholangiopancreatography: results of a nationwide Austrian benchmarking project. Endoscopy 2008;40:625-630.

28. Kalaitzakis E, Toth E. Hospital volume status is related to technical failure and all-cause mortality following ERCP for benign disease. Dig Dis Sci 2015;60:1793-1800. 